\title{
Prostatic Artery Embolization for Benign Prostatic Hyperplasia-A Primer for Interventional Radiologists
}

\author{
Tiago Bilhim ${ }^{1}$ Nuno V. Costa ${ }^{1}$ Daniel Torres ${ }^{1}$ D \\ ${ }^{1}$ Angiography Unit, NOVA Medical School, Faculdade de Ciências \\ Médicas, Universidade Nova de Lisboa, Saint Louis Hospital, Portugal \\ and Centro Hospitalar Universitário de Lisboa Central (CHULC), \\ Lisbon, Portugal \\ Arab J Intervent Radiol 2021;5:60-67.
}

\begin{abstract}
Address for correspondence Tiago C. A. de F. Bilhim, MD, PhD, EBIR, Angiography Unit, NOVA Medical School, Faculdade de Ciências Médicas, Universidade Nova de Lisboa, Saint Louis Hospital, Centro Hospitalar Universitário de Lisboa Central (CHULC), Rua Luz Soriano, 182, 1200-249 Lisbon, Portugal (e-mail: tiagobilhim@hotmail.com).
\end{abstract}

\begin{abstract}
Keywords

- prostatic artery embolization

- prostate embolization

- benign prostatic hyperplasia

- lower urinary tract symptoms

Male patients over 50 years with lower urinary tract symptoms (LUTS) and benign prostatic hyperplasia (BPH) are potential candidates for prostatic artery embolization (PAE). PAE is not a perfect fit for all BPH patients. Careful pre- and postpostprocedural evaluation/consultation with correct selection of patients should be tailored on an individual basis. Evaluated parameters include the following: LUTS severity quantification with validated questionnaires as the international prostate symptom score (IPSS) and quality of life (QoL), erectile and ejaculatory evaluation with validated questionnaires, blood tests including full blood count, coagulation profile, renal function and total/free prostatespecific antigen (PSA), prostate volume measured by multiparametric magnetic resonance (mpMR) of the prostate and/or transrectal ultrasound, uroflowmetry measuring the peak urinary flowrate (Qmax), and postvoid residual urine (PVR). Correct arterial anatomy identification with either computed tomography (CT) angiography, MR angiography, or intraprocedural cone-beam $C T$ (CBCT) are suggested for a confident procedure and avoiding potential complications. The minimally invasive nature of PAE with a faster recovery, preserving the sexual function, and comparable results to standard prostatic surgery make the procedure an attractive choice for many male patients suffering with this condition. Patients should be informed about the potential for higher retreatment rates and shorter duration of treatment effect when compared with standard prostatic surgery. In this comprehensive review, we provide an updated toolbox for all interventional radiologists interested in the PAE practice for patients with BPH. We explain how to evaluate patients during consultation before and after PAE, describe the preprocedural imaging required, explain the technique, and narrate how to optimize outcomes. Finally, we review the level of evidence of PAE for BPH.
\end{abstract}

\section{Preprocedural Patient Consultation}

Male patients over 50 years of age, presenting for consultation before prostatic artery embolization (PAE), usually have bothersome lower urinary tract symptoms (LUTS) and an enlarged prostate gland. Less frequently they can present with acute urinary retention (AUR) requiring a bladder catheter or gross hematuria. ${ }^{1}$ The most frequent cause for published online February 7, 2022
DOI https://doi.org/ 10.1055/s-0041-1739302. ISSN 2542-7075. (c) 2022. The Pan Arab Interventional Radiology Society. All rights reserved.

This is an open access article published by Thieme under the terms of the Creative Commons Attribution-NonDerivative-NonCommercial-License, permitting copying and reproduction so long as the original work is given appropriate credit. Contents may not be used for commercial purposes, or adapted, remixed, transformed or built upon. (https://creativecommons.org/ licenses/by-nc-nd/4.0/)

Thieme Medical and Scientific Publishers Pvt. Ltd., A-12, 2nd Floor, Sector 2, Noida-201301 UP, India 
LUTS and AUR in male patients over 50 years of age is benign prostatic hyperplasia (BPH) or benign prostatic enlargement (BPE). However, other causes may be present such as bladder dysfunction, urethral stricture, bladder stones or diverticula, prostate, and bladder cancer. ${ }^{2,3}$ Thus, preprocedural patient evaluation is essential to make sure that the correct diagnosis is made to optimize the treatment approach. For patients with gross hematuria, it is essential to perform a renal ultrasound to exclude renal cancer and a bladder ultrasound and/or cystoscopy to rule out a bladder tumor. Only, after excluding these causes, a prostatic origin can be assumed. ${ }^{3}$

The international prostate symptom score (IPSS) is a validated questionnaire that allows for confident quantification on the severity of LUTS before and after a prostatic intervention. The IPSS consists of seven questions, four for obstructive/voiding LUTS (hesitancy, weak stream, poor emptying, straining, prolonged micturition, and dribbling) and three for irritative/storage LUTS (frequency, urgency, urge incontinence, and nocturia) with a final question pertaining the overall quality of life (QoL) related to the LUTS. These seven questions are rated on a scale of 1 to 5 , providing and overall score of 0 to 35 points. The QoL question is graded on a scale of 0 to 6 ( 0 being delighted and 6 as terrible). Based on the overall IPSS score, LUTS are divided into mild (0-7 points), moderate (8-19 points), and severe (20-35 points). However, it is the QoL question that often dictates management. When the QoL is equal or greater than 4 , the patient is dissatisfied with the LUTS, and frequently requires an invasive treatment approach. ${ }^{1-3}$ It is important to stress that the IPSS/QoL allows for quantification of LUTS, but it does not allow for a confident diagnosis on the causes of LUTS. So, laboratory and imaging studies are needed to exclude other causes than BPH. Before PAE, to exclude prostatic malignancy, a digital rectal examination, blood tests with measurement of the prostate-specific antigen (PSA), and prostate multiparametric magnetic resonance ( $\mathrm{mpMR}$ ) might be useful tools. ${ }^{1}$

Another relevant point to consider is the severity of bladder outlet obstruction (BOO). Patients frequently are not bothered by BOO because it does not have an immediate impact on the QoL. However, BOO is critical when assessing the potential for complications from the progression of $\mathrm{BPH}$, such as AUR, bladder stones, or diverticula and obstructive uropathy. Postvoid residual urine (PVR) is an easy test using ultrasound of the bladder after urination that allows for confident screening of severe BOO. It should be included in the evaluation of all male patients with LUTS. Uroflowmetry is another easy and accessible noninvasive tool to assess BOO. It provides the peak urinary flowrate (Qmax) that should be quantified before and after a prostatic intervention, as it provides an indirect measure on the severity of BOO caused by BPH. A normal Qmax is > than $15 \mathrm{~mL} / \mathrm{s}$ with values under $12 \mathrm{~mL} / \mathrm{s}$ suggestive of BOO or underactive bladder. Invasive urodynamic studies can be used when BOO due to BPH is doubtful such as in young patients ( $<50$ years of age) with small prostate volumes $\left(<30 \mathrm{~cm}^{3}\right)$, predominately irritative LUTS, high Qmax (>15 mL/s), very high PVR $\left(>300 \mathrm{~cm}^{3}\right)$, or when a clinical suspicion of neurogenic bladder (diabetic patients and neurological diseases) exists. These invasive urodynamic studies can be very helpful as they differentiate LUTS from prostatic obstruction from LUTS due to bladder under- or hyperactivity. Bladder underactivity can be suspected when the Qmax is less than $12 \mathrm{~mL} / \mathrm{s}$ in diabetic patients or patients suffering from neurological diseases, when the PVR is $>300 \mathrm{~cm}^{3}$, when multiple large bladder diverticula are present, or when bilateral hydronephrosis is seen. Bladder hyperactivity can be suspected in young patients with Qmax $>15 \mathrm{~mL} / \mathrm{s}$ and predominately irritative LUTS. It should be stressed; however, that bladder hyperactivity frequently coexists with $\mathrm{BOO}$ due to $\mathrm{BPH} .^{2,3}$

When dealing with LUTS patients in consultation prior to PAE, one of the key aspects is managing expectations. Patients will enquire about mostly three main things as follows: (1) is it painful/complications, (2) recovery period, and/or (3) effectiveness. Regarding the procedure, it is very well tolerated. PAE is a painless procedure that is performed under 2 hours and can be performed under an outpatient setting. Adverse events are present in less than $3 \%$ of patients, even though frequency and a burning sensation during urination might be felt by up to $50 \%$ of treated patients in the first 1 or 2 days. The recovery is almost immediate, as patients can return to normal daily activities after 2 or 3 days. The effectiveness is around $80 \%$, which means that $80 \%$ of patients will have their LUTS significantly improved after PAE for the following 3 to 5 years. Data are still scarce to inform on longer follow-up above 5 years. This also means that up to $20 \%$ of treated patients may not improve or do not improve as much the LUTS as they wanted. This might mean that these patients may require prostatic medication or surgery soon. When dealing with AUR patients, we usually inform a $90 \%$ chance of success on spontaneous urination and freedom from bladder catheter within 2 weeks and 1 month after PAE. ${ }^{1}$

Finally, when dealing with male patients with LUTS, erectile and ejaculatory function are also discussed. One of the main advantages of PAE when compared with prostatic surgery is the fact that it allows a painless, almost immediate recovery preserving both erectile and ejaculatory functions. It should be stressed, however, that PAE is not a procedure to improve erectile and ejaculatory functions. Prostatic medication is another topic to debate. Most patients presenting to consultation have already been treated with $\alpha$-blockers and/or 5 - $\alpha$-reductase inhibitors. The $\alpha$-blockers have an immediate effect and washout and significantly improve LUTS, whereas 5 - $\alpha$-reductase inhibitors reduce prostate volume (25-30\%) and PSA (up to 50\%) with minimal effect on LUTS, with a longer time to reach effect and washout (3-6 months). These $\alpha$-blockers are first-line therapy in virtually all LUTS patients, whereas $5-\alpha$-reductase inhibitors might be recommended in high-risk patients (prostate volume $>40$ $\mathrm{cm}^{3}$ ) to prevent disease progression. Also, $\alpha$-blockers may cause orthostatic hypotension or retrograde ejaculation, whereas 5 - $\alpha$-reductase inhibitors may lead to gynecomastia, reduced libido, and erectile dysfunction. Most patients treated with PAE can discontinue all prostatic medication after the embolization. Anticholinergic medication is also 
used when irritative LUTS are predominant, and a hyperactive bladder is suspected, provided that $\mathrm{Qmax}>12 \mathrm{~mL} / \mathrm{s}^{1-3}$

To summarize, when consulting patients before PAE the followings can be considered: LUTS versus AUR versus gross hematuria, for LUTS patients evaluate if voiding versus storage symptoms predominate, inquire about prostatic medication, and erectile/ejaculatory expectations. At this point, validated questionnaires are very useful such as IPSS/QoL, international index of erectile function (IIEF-5 questions version), and ejaculatory function questionnaire. Blood tests including full blood count, coagulation profile, renal function, and total/free PSA values should be obtained. We recommend mpMR of the prostate before PAE. Uroflowmetry measuring the Qmax and PVR should also be used. Renal and bladder ultrasound are also frequently performed. Follow-up after PAE should be at 1 month, 6 months, and then yearly with IPSS/QoL, IIEF-5, PSA, prostate volume measured by transrectal ultrasound, Qmax, and PVR. Cystoscopy might be needed when hematuria is present, when the prostatic obstruction is doubtful, or when bladder cancer is suspected. Invasive urodynamic studies are required in specific situations as outlined above.

\section{Imaging Planning for Prostatic Artery Embolization}

As discussed above, some imaging studies are usually required before PAE. Bladder ultrasound to measure the PVR and study the bladder should be used routinely. ${ }^{3}$ Prostate volume measurement and zonal anatomy of the prostate could be done with transrectal ultrasound or mpMR of the prostate. ${ }^{4}$ mpMR has advantages over ultrasound, as it allows for confident identification of prostate cancer, better zonal volumetry and estimation of the intravesical prostatic protrusion, detailed study of the bladder wall, and lumen and can also be used for vascular assessment of the pelvic arteries. However, mpMR is more expensive, time consuming, and less available than prostatic ultrasound. As such, mpMR of the prostate can be used at baseline, whereas transrectal ultrasound can be used after PAE to assess prostate volume. ${ }^{4}$ Another aspect relevant for PAE planning relies on the vascular mapping of the pelvic and prostatic arteries. One approach has been using computed tomography angiography (CTA) prior to PAE to study the anatomy of the pelvic and prostatic arteries. ${ }^{5}$ CTA prior to PAE allows confident identification of the prostatic arteries and helps plan the procedure. ${ }^{5,6}$ CTA can also be used to predict the level of difficulty of the PAE procedure and predict technical outcomes. ${ }^{7}$ To achieve predictable and confident identification of the prostatic arteries, the CTA protocols should be adjusted: contrast used should have concentrations 350 to $400 \mathrm{mg} \mathrm{I} / \mathrm{mL}$ with a volume of 100 to $120 \mathrm{~mL}$, flowrate of $4 \mathrm{~mL} / \mathrm{s}$, saline flushing $(40 \mathrm{~mL})$ at the same rate; acquisition times for the pelvis should be 10 to 15 seconds (too fast or too slow can affect correct prostatic artery opacification); delay usually 16 to 20 seconds based on bolus tracking in the aorta (200 Hounsfield's Units level of threshold), leading to approximately 30 to 35 seconds from contrast injection until the end of acquisition. ${ }^{5}$ The use of $0.5 \mathrm{mg}$ sublingual nitroglycerin has also shown to be useful to improve prostatic artery opacification. 5,6

Modern angiography machines have flat panel detectors that allow the use of rotational three-dimensional (3D) conebeam CT (CBCT) scans besides the two-dimensional (2D) conventional digital subtraction angiographies (DSA). The use of CBCT is very important for PAE and can be used in following two scenarios: (1) to map the pelvic arteries and identify the prostatic arteries, and (2) to certify correct microcatheter position within the prostatic arteries and prostatic coverage and exclude anastomoses. CBCT has shown to have similar accuracy to CTA in identifying the prostatic arteries with lower radiation exposure and contrast volume used. ${ }^{8}$ However, CBCT can only be performed with the patient already in the angiosuite during PAE; CBCT does not allow for preprocedural vascular assessment. CBCT allows for the acquisition of $3 \mathrm{D}$ datasets that can be used with dedicated software's, with automatic feeder detection. These software's automatically identify the arteries feeding the prostate and provide an overlay with 2D fluoroscopy and the $3 \mathrm{D}$ roadmaps allowing direct guidance into the prostate, obviating extensive knowledge of the pelvic arterial anatomy. ${ }^{9}$ More recently, CBCT has shown to be superior to DSA in detecting the prostatic arteries, allowing for a reduced number of DSA runs with an overall decrease in radiation exposure. $^{10}$

CBCT injection protocols should be adjusted according to rotational scan time and catheter position. Injection of contrast should cover the whole scanning time plus 3 to 5 seconds. The injection should start before CBCT start (scan delay). Diluted contrast media/saline (50\%/50\%) can be used for CBCT. Some examples of CBCT injection protocols with a rotational scan of 10 seconds are as follows: with the catheter in the aorta: $35 \mathrm{~mL}, 3 \mathrm{~mL} / \mathrm{s}$, and scan delay for 4 seconds; with the catheter in the internal iliac artery: $20 \mathrm{~mL}, 1.5 \mathrm{~mL} / \mathrm{s}$, and scan delay for 4 seconds; and with the microcatheter in the prostatic artery: $8 \mathrm{~mL}, 0.5 \mathrm{~mL} / \mathrm{s}$, and scan delay for 3 seconds. CBCT with the catheter in the distal aorta allows roadmapping of the whole pelvic arteries, whereas in the internal iliac arteries only one pelvic side is studied. Both injections are used to map the pelvic arteries and identify the prostatic arteries. On the other hand, selective CBCT injections in the prostatic arteries are used to certify correct microcatheter position, exclude relevant anastomoses that could lead to nontarget embolization, and certify if more than one prostatic artery is present in each pelvic side. ${ }^{8-11}$ It has been shown that in up to $20 \%$ of patients, more than one prostatic artery feeding the central gland may be present on either pelvic side. ${ }^{5,11}$

Recently, MR angiography protocols with 3-Tesla scanners have shown high accuracy ( $>90 \%$ ) for detecting the prostatic arteries. $^{12}$ This has a huge advantage of assessing the prostate with mpMR and the pelvic arteries with MR within a single examination. This has led to a shift from preprocedural CTA towards MRA (-Fig. 1). With preprocedural MRA, procedural times, radiation exposure, and contrast volume used are significantly reduced during PAE. ${ }^{12}$ The MR 

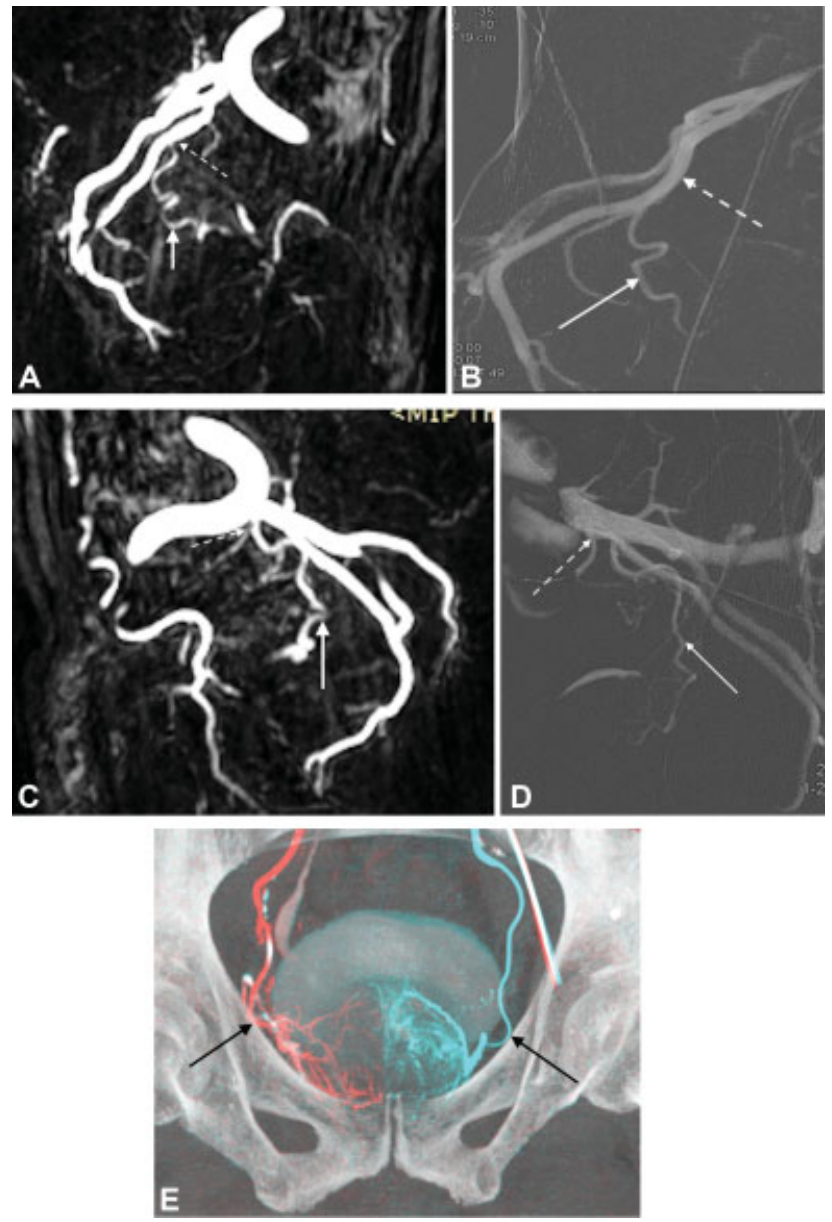

Fig. 1 (A) MRA 3D-oblique view of a maximum intensity projection reformat of the right internal iliac artery, depicting the prostatic artery (arrow) arising from the internal pudendal artery (dashed arrow). (B) DSA with ipsilateral anterior oblique view (35 degrees) of the right internal iliac artery, depicting the prostatic artery (arrow) arising from the internal pudendal artery (dashed arrow). (C) MRA 3Doblique view of a maximum intensity projection reformat of the left internal iliac artery, depicting the prostatic artery (arrow) arising from the superior vesical artery (dashed arrow). (D) DSA with ipsilateral anterior oblique view ( 35 degrees) of the left internal iliac artery, depicting the prostatic artery (arrow) arising from the superior vesical artery (dashed arrow). Note how closely the MRA and DSA images match, certifying the accuracy of preprocedural MRA to identify the prostatic arteries. (E) CBCT after selective prostatic artery catheterization with a microcatheter (arrows), in both pelvic sides, confirming correct location and excluding anastomoses that could lead to nontarget embolization. 3D, three dimensional; $C B C T$, cone-beam computed tomography; DSA, digital subtraction angiographies; MRA, magnetic resonance angiography.

scanners need to be 3 Tesla to be able to have acquisition times like CTA with the same image resolution, using surface phase array coils to scan the whole pelvis (including the distal aorta). It is possible to have voxels of 1 to $2 \mathrm{~mm}$ and perform volumetric scanning of the whole pelvis in 10 to 20 seconds. With MRA, the pelvis can be analyzed with and without subtraction. Injection protocols usually use 0.1 to $0.2 \mathrm{~mL}$ per $\mathrm{kg}$ of body weight, with a total of 16 to $25 \mathrm{~mL}$ of intravenous gadolinium, at a flowrate of $3 \mathrm{~mL} / \mathrm{s}$, followed by saline flushing $(2 \mathrm{~mL} / \mathrm{s})$. We adjusted a previous published protocol $^{12}$ to identify the prostatic arteries during MRA; after the administration of $0.2 \mathrm{~mL} / \mathrm{kg}$ of Dotarem (gadoteric acid; Guerbet, Roissy CdG, France) injected at $3 \mathrm{~mL} / \mathrm{s}$ using a power injector followed by $20 \mathrm{~mL}$ saline flush at the rate of $2 \mathrm{~mL} / \mathrm{s}$. MRA using 3D volumetric interpolated spoiled gradient echo was obtained with the following parameters: field of view extending from the iliac bifurcation to the common femoral arteries with $1.3-\mathrm{mm}$ slice thickness; matrix: $256 \times 100$ (voxel of $1.3 \mathrm{~mm} \times 0.9 \mathrm{~mm} \times 0.9 \mathrm{~mm}$ ); repitition time (TR): 7.9; echo time (TE): 3.6; and acquisition time of 22 seconds of a total of five phases ( $\mathbf{- V i d e o s} \mathbf{1}$ and $\mathbf{2}$ ).

\section{Video 1}

Axial MR angiography images depicting the pelvic and prostatic arteries without subtraction. MR, magnetic resonance. Online content including video sequences viewable at: https://www.thieme-connect.com/products/ ejournals/html/10.1055/s-0041-1739302.

\section{Video 2}

Axial MR angiography images depicting the pelvic and prostatic arteries with subtraction. MR, magnetic resonance. Online content including video sequences viewable at: https://www.thieme-connect.com/products/ ejournals/html/10.1055/s-0041-1739302.

\section{Technique}

PAE is all about anatomy, having the right tools and knowing how/when to use them. Starting with anatomy, it is important to have a clear idea on all major pelvic side branches, including the superior and inferior gluteal arteries, the internal pudendal, and the obturator arteries. With ipsilateral anterior oblique views, it is relatively easy to identify all these major pelvic vessels and thus identify the smaller side branches. The ipsilateral anterior oblique views help to separate all the major internal iliac artery side branches and correctly identify the vesical, prostatic, and rectal arteries. ${ }^{13,14}$ Most bladder arteries arise from the superior vesical artery that is always the first side branch of the anterior division of the internal iliac artery. The superior vesical artery should not be confused with the prostatic artery. The superior vesical artery has a straight trajectory forward and medially into the bladder, whereas the prostatic artery has a trajectory first caudally and only after that it runs medially (in a C or L trajectory), underneath the bladder. The prostatic artery frequently has a parallel trajectory to the internal pudendal artery and is usually the most tortuous pelvic vessel. After prostatic artery catheterization, the prostate arteries are seen overlying the pubic bone as seen on 2D fluoroscopic imaging, usually with horizontal branches. Vertical branches above the pubic bone should alert to the 
possibility of bladder or rectal branches, whereas underneath the pubic bone to penile branches. CBCT after selective catheterization of the prostatic arteries allows for certification of correct location and depiction of anastomoses that could lead to nontarget embolization. The most frequent prostatic artery origins include superior vesical, internal pudendal, anterior division of the internal iliac artery, and obturator artery (accounting for more than $75 \%$ of all origins). Less-frequent origins include accessory pudendal arteries, superior or inferior gluteal arteries, aberrant obturator arteries, and common prostate-rectal trunks. Relevant variants to be aware of encompass: accessory pudendal arteries and penile anastomoses that could lead to penile nontarget embolization, rectal branches that could lead to rectal ischemia and aberrant obturator arteries that are outside the pelvic region and could originate the prostatic arteries in up to $1 \%$ of pelvic sides. ${ }^{5,13-15}$ The superior vesical artery is the most challenging anatomy. When the prostatic artery arises from the superior vesical artery, it can be very challenging to navigate past the bladder branches. However, embolization of the whole superior vesical artery should not be performed, as it can lead to complications from severe bladder ischemia.

With preprocedural CTA or MRA or using CBCT of the pelvic arteries, it is possible to correctly identify the prostatic arteries and their origins. As such, there is no need for DSA angiographic runs that may be responsible for up to $75 \%$ of radiation exposure during PAE. ${ }^{16}$ After catheterization of the internal iliac artery, ipsilateral anterior oblique views (35 to 45 degrees) are performed with caudal-cranial (-10 degrees) angulations. These oblique views help to navigate inside the pelvic branches but increase the radiation exposure. So, avoiding DSA runs and using roadmap instead, may help to reduce the radiation exposure to patients. Under the roadmap guidance with steep oblique views, it is possible to catheterize the prostatic arteries identified with the pelvic CBCT, CTA, or MRA. Whenever possible, DSA runs to map the pelvic arteries and search for the prostatic arteries should be avoided. After selective prostatic artery catheterization, CBCT can be performed to assess correct location and exclude relevant anastomoses (-Videos 3 and 4; available in the online version). After embolization of the prostatic arteries, complete stasis should be achieved. The vascular access for PAE can be radial or femoral. Specific requirements are needed for a safe radial approach (radial artery $>2 \mathrm{~mm}$ in diameter; Barbeau's test A, B, or C; patient age $<75$ years and height $<1.85 \mathrm{~m}$ ). Patients prefer radial access to femoral access, as they can ambulate immediately after PAE and use the toilets as needed. ${ }^{13}$ Radial access for PAE has been shown to be safe and effective. ${ }^{17}$ When using a radial access, dedicated radial sheaths, adequate catheters, and catheter lengths should be used. ${ }^{18}$

\section{Video 3}

Axial CBCT images after selective prostatic artery catheterization with a microcatheter in both pelvic sides, confirming correct location and excluding anastomoses that could lead to nontarget embolization. CBCT, cone-beam computed tomography. Online content including video sequences viewable at: https:// www.thieme-connect.com/products/ejournals/html/ 10.1055/s-0041-1739302.

\section{Video 4}

Video depicting a PAE procedure on the right pelvic side from a left radial approach. PAE, prostatic artery embolization. Online content including video sequences viewable at: https://www.thieme-connect.com/products/ ejournals/html/10.1055/s-0041-1739302.

As required tools for PAE, catheters, microcatheters, and microwire preferences vary among interventional radiologists. $^{13-18}$ Most would agree that 2.0- to 2.4-F microcatheters with preshaped swan-neck curves at the tip (Progreat Lambda, Terumo [Tokyo, Japan], Maestro or Pursue, Merit Medical Systems, Inc. [South Jordan, UT, USA]; Direxion, Boston Scientific Corporation [Marlborough, MA, USA]) are the first-line option for PAE. Having these angulations at the microcatheter tip allows for navigation with torque capability, even without the need for microwires. In up to $60 \%$ of PAE procedures, we can only use microcatheters without microwires. It is also important to have multiple choices when it comes to microwire selection; most frequently used ones include 0.014- to 0.016inch Glidewire GT (double-angled or 90-degree angled, Terumo, Tokyo, Japan), Fathom (shapeable, Boston Scientific Corporation, Marlborough, MA, USA), Asahi Meister (shapeable, Asahi Intecc USA, Inc.), Hi-Torque BMW wire (Abbott, Chicago, IL, USA), and Synchro (Stryker, Kalamazoo, MI, USA). With the most challenging PAE procedures, in older patients, with severe arterial tortuosity and atherosclerosis and prostatic arteries arising from the superior vesical artery, having steerable microcatheters can be very useful (SwiftNinja, Merit Medical Systems, Inc., South Jordan, UT, USA).

\section{Optimizing Outcomes}

Outcomes can be optimized through technique and patient selection. It is already proven that bilateral PAE provides better outcomes than unilateral PAE. ${ }^{19,20}$ Thus, physician expertise is needed, as it has a direct impact on technical outcomes. ${ }^{21,22}$ Coil protection during PAE might be required in up to $25 \%$ of patients before embolization ${ }^{21}$ to exclude anastomoses that could lead to complications from nontarget embolization. The use of protective coils prior to embolization increases the radiation exposure but allows for a safe and effective PAE. ${ }^{21,23}$ The paradox as to the best embolic agent and size for PAE remains, with conflicting evidence from existing literature. Currently used embolic agents include 100 - to $300-\mu \mathrm{m}$ polyvinyl alcohol (PVA) particles (Bearing nsPVA, Merit Medical Systems, Inc.; Contour, Boston Scientific Corporation) and 
spherical embolic agents such as spherical PVA (300-500 $\mu \mathrm{m}$ Bead Block, Boston Scientific Corporation), 100- to 300- $\mu \mathrm{m}$ and/or 300- to 500- $\mu \mathrm{m}$ trisacryl gelatin microspheres (Embosphere, Merit Medical Systems, Inc.), 250- $\mu \mathrm{m}$ and/or 400- $\mu \mathrm{m}$ polyzene-coated hydrogel microspheres (Embozene, Varian Medical Systems), and $250-\mu \mathrm{m}$ and/or $400-\mu \mathrm{m}$ polyethylene glycol microspheres (HydroPearl, Terumo Interventional). There is conflicting evidence regarding the use of smaller particles and the potential to have better outcomes or longer treatment effects. It has been proven that smaller spherical embolic agents may lead to more adverse events, even though the safety profile is the same for PVA particles regardless the size. ${ }^{21,24-28}$ As such, one could recommend using 100 to $300 \mu \mathrm{m}$ PVA particles or $400-\mu \mathrm{m}$ or 300 - to $500-\mu \mathrm{m}$ spherical embolic agents.

As for optimizing patient selection, there is conflicting evidence regarding baseline patient factors that may predict a good or bad clinical outcome. ${ }^{29}$ Patients under AUR seem to respond very well to PAE. Younger patients seem to perform better than older patients and the potential for larger prostates to have better outcomes is highly controversial, even though the ratio of transitional prostate volume/whole prostate volume $>50 \%$ and the presence of large $(>1 \mathrm{~cm})$ central gland adenomas predict better outcomes. ${ }^{29}$ The presence of pedunculated median lobes has proven to lead to worse clinical outcomes after PAE. ${ }^{30}$ Post-PAE identified predictors of clinical outcome include blood tests with 24hour post-PAE PSA and C-reactive protein levels, MR detected prostate infarction, and prostate volume reduction within the first month post-PAE.

\section{Level of Evidence}

The safety and efficacy of PAE has been shown in multiple phase-II trials. In a recent meta-analysis ${ }^{31}$ including 1,046 patients from 10 studies with a mean follow-up of 12 months, PAE induced a mean IPSS improvement of 16.2 points, a mean QoL improvement of 3.0 points, and a mean prostate volume reduction of $20.3 \mathrm{~cm}^{3}$ (25\%). These improvements remained statistically significant throughout 3 years. Minor adverse events, including the postembolization syndrome with transient dysuria in $10 \%$ and increased urinary frequency in $16 \%$ of patients were reported. Major adverse events were reported in three patients (0.3\%): one bladder ischemia, one urinary tract infection, and one persistent perineal pain.

More recently, a systematic review analyzed six trials comparing PAE with transurethral resection of the prostate (TURP) in 598 patients. ${ }^{32-37}$ TURP provided a significantly higher increase of the Qmax (mean difference of $5.02 \mathrm{~mL} / \mathrm{s}$ ), greater prostate volume reduction (mean difference $=15.59$ $\mathrm{mL}$ ) and greater PSA reduction (mean difference $=1.02$ $\mathrm{ng} / \mathrm{mL}$ ). No significant differences between PAE and TURP were noted for the IPSS/QoL scores, IIEF-5 scores and PVR. There were significantly less adverse events with PAE (39.0 vs. $77.7 \%$ ) and shorter hospitalization times with PAE (mean difference $=-1.94$ days). PAE had significantly longer procedural times (mean difference $=51.43$ minutes). Another recent meta-analysis ${ }^{38}$ of comparative studies includ- ing PAE, analyzed six randomized clinical trials against TURP and two nonrandomized comparative trials against prostatic surgery and one comparative trial against a sham procedure. ${ }^{39}$ Little to no difference in IPSS and QoL improvements when PAE is compared with prostatic surgery. However, PAE may increase retreatments rates (risk ratio $=3.64$ in short-term and risk-ratio of 1.51 in long-term). PAE may reduce the occurrence of ejaculatory disorders (risk ratio of 0.51 ). The need for more studies reporting long-term data after PAE was highlighted. ${ }^{38}$ Long-term data after PAE have shown the potential for a $20 \%$ retreatment rate at 2 years, with clinical success rates of approximately $75 \%$ after 3 years. ${ }^{40-43}$ Repeat PAE may be an option especially for those patients who initially improved after embolization but had relapsing symptoms. ${ }^{44}$ Most interventional radiology guidelines recommend PAE as a valid treatment option for BPH patients, ${ }^{45,46}$ as well as some national ${ }^{47}$ guidelines and European urology guidelines. ${ }^{48}$

\section{Conclusion}

PAE is a minimally invasive treatment option for BPH patients with symptomatic improvements comparable to standard prostatic surgery. The key aspects for the preprocedural patient consultation and evaluation, imaging planning for PAE, technique, and optimizing outcomes are revised. The minimally invasive nature of PAE with a faster recovery, preserving the sexual function is very attractive to patients. However, patients should be informed about the potential risks of higher retreatment rates with PAE and shorter duration of treatment effect when compared with standard prostatic surgery.

\section{Conflicts of Interest}

T.C.A.F.B.: Speaker fees for Terumo, Merit, Philips, Cook, stockholder for EmbolX. N.V.C.: Speaker fees for Terumo, Merit, and Bial. All the other authors report no conflict of interest.

\section{References}

1 A Pereira J,Bilhim T, Duarte M, Rio Tinto H, Fernandes L, Martins Pisco J. Patient selection and counseling before prostatic arterial embolization. Tech Vasc Interv Radiol 2012;15 (04):270-275

2 D'Silva KA, Dahm P, Wong CL. Does this man with lower urinary tract symptoms have bladder outlet obstruction?: the rational clinical examination: a systematic review JAMA 2014;312(05): 535-542

3 Gratzke C, Bachmann A, Descazeaud A, et al. EAU guidelines on the assessment of non-neurogenic male lower urinary tract symptoms including benign prostatic obstruction. Eur Urol 2015;67 (06):1099-1109

4 Dias JL, Bilhim T. Modern imaging and image-guided treatments of the prostate gland: MR and ablation for cancer and prostatic artery embolization for benign prostatic hyperplasia. BJR Open 2019;1(01):20190019

5 Bilhim T, Pisco JM, Rio Tinto H, et al. Prostatic arterial supply: anatomic and imaging findings relevant for selective arterial embolization. J Vasc Interv Radiol 2012;23(11):1403-1415 
6 Little MW, Macdonald AC, Boardman P, et al. Effects of sublingual glyceryl trinitrate administration on the quality of preprocedure CT angiography performed to plan prostate artery embolization. J Vasc Interv Radiol 2018;29(02):225-228

7 Lintin L, Barge T, Boardman P, Tong G, Tapping C. Predictors of technical outcome for prostatic artery embolisation using preprocedural CT angiography. Eur Radiol 2021;31(03):1308-1315

8 Desai H, Yu H, Ohana E, Gunnell ET, Kim J, Isaacson A. Comparative analysis of cone-beam CT angiogram and conventional CT angiogram for prostatic artery identification prior to embolization. J Vasc Interv Radiol 2018;29(02):229-232

9 Schott P, Katoh M, Fischer N, Freyhardt P. Radiation dose in prostatic artery embolization using cone-beam CT and 3D roadmap software. J Vasc Interv Radiol 2019;30(09):1452-1458

10 Schnapauff D, Maxeiner A, Wieners G, et al. Semi-automatic prostatic artery detection using cone-beam $\mathrm{CT}$ during prostatic arterial embolization. Acta Radiol 2020;61(08):1116-1124

11 DeMeritt JS, Wajswol E, Wattamwar A, Osiason A, ChervoniKnapp T, Zamudio S. Duplicated prostate artery central gland blood supply: a retrospective analysis and classification system. J Vasc Interv Radiol 2018;29(11):1595-1600.e9

12 Zhang JL, Wang MQ Shen YG, et al. Effectiveness of contrastenhanced MR angiography for visualization of the prostatic artery prior to prostatic arterial embolization. Radiology 2019;291(02): 370-378

13 Bilhim T. Prostatic artery embolization for benign prostatic hyperplasia: a 10-year update. Endovasc Today 2020;19:40-44

14 Bilhim T, Pereira JA, Fernandes L, Rio Tinto H, Pisco JM. Angiographic anatomy of the male pelvic arteries. AJR Am J Roentgenol 2014;203(04):W373-82

15 Picel AC, Hsieh TC, Shapiro RM, Vezeridis AM, Isaacson AJ. Prostatic artery embolization for benign prostatic hyperplasia: patient evaluation, anatomy, and technique for successful treatment. Radiographics 2019;39(05):1526-1548

16 Andrade G, Khoury HJ, Garzón WJ, et al. Radiation exposure of patients and interventional radiologists during prostatic artery embolization: a prospective single-operator study. J Vasc Interv Radiol 2017;28(04):517-521

17 Bhatia S, Harward SH, Sinha VK, Narayanan G. Prostate artery embolization via transradial or transulnar versus transfemoral arterial access: technical results. J Vasc Interv Radiol 2017;28(06): 898-905

18 Bilhim T, Iezzi R, Guimaraes M. Perspectives on radial access applications: embolization therapies. Endovasc Today 2020; 19:34-37

19 Bilhim T, Pisco J, Rio Tinto H, et al. Unilateral versus bilateral prostatic arterial embolization for lower urinary tract symptoms in patients with prostate enlargement. Cardiovasc Intervent Radiol 2013;36(02):403-411

20 Bilhim T, Pisco J, Pereira JA, et al. Predictors of clinical outcome after prostate artery embolization with spherical and nonspherical polyvinyl alcohol particles in patients with benign prostatic hyperplasia. Radiology 2016;281(01):289-300

21 Hacking N, Vigneswaran G, Maclean D, et al. Technical and imaging outcomes from the UK Registry of Prostate Artery Embolization (UK-ROPE) study: focusing on predictors of clinical success. Cardiovasc Intervent Radiol 2019;42(05): 666-676

22 Enderlein GF, Lehmann T, von Rundstedt FC, et al. Prostatic artery embolization-anatomic predictors of technical outcomes. J Vasc Interv Radiol 2020;31(03):378-387

23 Bhatia S, Sinha V, Bordegaray M, Kably I, Harward S, Narayanan G. Role of coil embolization during prostatic artery embolization: incidence, indications, and safety profile ${ }^{\hat{t}}$. J Vasc Interv Radiol 2017;28(05):656-664.e3

24 Bilhim T, Pisco J, Campos Pinheiro L, et al. Does polyvinyl alcohol particle size change the outcome of prostatic arterial embolization for benign prostatic hyperplasia? Results from a single- center randomized prospective study. J Vasc Interv Radiol 2013;24(11):1595-602.e1

25 Wang MQ Zhang JL, Xin HN, et al. Comparison of clinical outcomes of prostatic artery embolization with $50-\mu \mathrm{m}$ plus $100-\mu \mathrm{m}$ polyvinyl alcohol (PVA) particles versus $100-\mu \mathrm{m}$ pva particles alone: a prospective randomized trial. J Vasc Interv Radiol 2018; 29(12):1694-1702

26 Abt D, Müllhaupt G, Mordasini L, et al. Outcome prediction of prostatic artery embolization: post hoc analysis of a randomized, open-label, non-inferiority trial. BJU Int 2019;124(01):134-144

27 Gonçalves OM, Carnevale FC, Moreira AM, Antunes AA, Rodrigues VC, Srougi M. Comparative study using $100-300$ versus $300-$ $500 \mu \mathrm{m}$ microspheres for symptomatic patients due to enlargedBPH prostates. Cardiovasc Intervent Radiol 2016;39(10): 1372-1378

28 Torres D, Costa NV, Pisco J, Pinheiro LC, Oliveira AG, Bilhim T. Prostatic artery embolization for benign prostatic hyperplasia: prospective randomized trial of $100-300 \mu \mathrm{m}$ versus $300-500 \mu \mathrm{m}$ versus 100 - to $300-\mu \mathrm{m}+300$ - to $500-\mu \mathrm{m}$ Embospheres. J Vasc Interv Radiol 2019;30(05):638-644

29 Sun F, Lucas-Cava V, Sánchez-Margallo FM. Clinical predictive factors in prostatic artery embolization for symptomatic benign prostatic hyperplasia: a comprehensive review. Transl Androl Urol 2020;9(04):1754-1768

$30 \mathrm{Yu}$ SCH, Cho CCM, Hung EHY, et al. Thickness-to-height ratio of intravesical prostatic protrusion predicts the clinical outcome and morbidity of prostatic artery embolization for benign prostatic hyperplasia. J Vasc Interv Radiol 2019;30(11):1807-1816

31 Malling B, Røder MA, Brasso K, Forman J, Taudorf M, Lönn L. Prostate artery embolisation for benign prostatic hyperplasia: a systematic review and meta-analysis. Eur Radiol 2019;29(01): 287-298

32 Knight GM, Talwar A, Salem R, Mouli S. Systematic review and meta-analysis comparing prostatic artery embolization to goldstandard transurethral resection of the prostate for benign prostatic hyperplasia. Cardiovasc Intervent Radiol 2021;44(02): 183-193

33 Insausti I, Sáez de Ocáriz A, Galbete A, et al. Randomized comparison of prostatic artery embolization versus transurethral resection of the prostate for treatment of benign prostatichyperplasia.J Vasc Interv Radiol 2020;31(06):882-890

34 Abt D, Hechelhammer L, Müllhaupt G, et al. Comparison of prostatic artery embolisation (PAE) versus transurethral resection of the prostate (TURP) for benign prostatic hyperplasia: randomised, open label, non-inferiority trial. BMJ 2018;361: k2338

35 Carnevale FC, Iscaife A, Yoshinaga EM, Moreira AM, Antunes AA Srougi M. Transurethral resection of the prostate (turp) versus original and perfected prostate artery embolization (pae) due to benign prostatic hyperplasia (bph): preliminary results of a single center, prospective. Urodyn-Controll Anal. Cardiovasc Intervent Radiol 2016;39(01):44-52

36 Gao YA, Huang Y, Zhang R, et al. Benign prostatic hyperplasia: prostatic arterial embolization versus transurethral resection of the prostate-a prospective, randomized, and controlled clinical trial. Radiology 2014;270(03):920-928

37 Ray AF, Powell J, Speakman MJ, et al. Efficacy and safety of prostate artery embolization for benign prostatic hyperplasia: an observational study and propensity-matched comparison with transurethral resection of the prostate (the UK-ROPE study). BJU Int 2018;122(02):270-282

38 Jung JH, McCutcheon KA, Borofsky M, et al. Prostatic arterial embolization for the treatment of lower urinary tract symptoms in men with benign prostatic hyperplasia. Cochrane Database Syst Rev 2020;12:CD012867

39 Pisco JM, Bilhim T, Costa NV, et al. Randomised clinical trial of prostatic artery embolisation versus a sham procedure for benign prostatic hyperplasia. Eur Urol 2020;77(03):354-362 
40 Pisco JM, Bilhim T, Pinheiro LC, et al. Medium- and long-term outcome of prostate artery embolization for patients with benign prostatic hyperplasia: results in 630 patients. J Vasc Interv Radiol 2016;27(08):1115-1122

41 Carnevale FC, Moreira AM, de Assis AM, et al. Prostatic artery embolization for the treatment of lower urinary tract symptoms due to benign prostatic hyperplasia: 10 years' experience. Radiology 2020;296(02):444-451

42 Lin YT, Pereira H, Pellerin O, Déan C, Thiounn N, Sapoval M. Fouryear impact of voiding and storage symptoms in patients with benign prostatic hyperplasia treated with prostatic artery embolization. J Vasc Interv Radiol 2020;31(09):1460-1466

43 Abt D, Müllhaupt G, Hechelhammer L, et al. Prostatic artery embolisation versus transurethral resection of the prostate for benign prostatic hyperplasia: 2-yr outcomes of a randomised, open-label, single-centre trial. Eur Urol 2021;80(01):34-42

44 Costa NV, Torres D, Pisco J, et al. Repeat prostatic artery embolization for patients with benign prostatic hyperplasia. J Vasc Interv Radiol 2020;31(08):1272-1280

45 Cornelis FH, Bilhim T, Hacking N, Sapoval M, Tapping CR, Carnevale FC. CIRSE standards of practice on prostatic artery embolisation. Cardiovasc Intervent Radiol 2020;43(02):176-185
46 McWilliams JP, Bilhim TA, Carnevale FC, et al. Society of Interventional Radiology Multisociety Consensus position statement on prostatic artery embolization for treatment of lower urinary tract symptoms attributed to benign prostatic hyperplasia: from the Society of Interventional Radiology, the Cardiovascular and Interventional Radiological Society of Europe, Société Française de Radiologie, and the British Society of Interventional Radiology: Endorsed by the Asia Pacific Society of Cardiovascular and Interventional Radiology, Canadian Association for Interventional Radiology, Chinese College of Interventionalists, Interventional Radiology Society of Australasia, Japanese Society of Interventional Radiology, and Korean Society of Interventional Radiology. J Vasc Interv Radiol 2019;30(05):627-637.e1

47 NICE Guidance - Prostate artery embolisation for lower urinary tract symptoms caused by benign prostatic hyperplasia: NICE (2018) Prostate artery embolisation for lower urinary tract symptoms caused by benign prostatic hyperplasia. BJU Int 2018;122(01):11-12

48 The EAU Annual Congress Milan 2021. Accessed July 8, 2021 at: https://eaucongress.uroweb.org/info-centre/ 